\title{
Investigating complex cardiovascular dynamics during hedonic olfactory elicitation
}

\author{
M. Nardelli ${ }^{1}$, A. Lanata $^{1}$, F. di Francesco ${ }^{2}$, E. P. Scilingo ${ }^{1}$
}

\begin{abstract}
Olfactory sense plays a fundamental role in human behaviour such as emotion regulation, memory formation, risk evaluation, and food appreciation. However, the effects of hedonic olfactory stimuli on autonomic nervous system response are still unclear. In this study we investigate heartbeat complexity in a group of 32 healthy subjects, after the elicitation with three odorants commonly considered to be pleasant, neutral, and unpleasant. Results show significant differences in complexity of cardiovascular system dynamics when subjects smell pleasant and unpleasant odors (p-value $<0.05)$.
\end{abstract}

\section{INTRODUCTION}

Perception of emotional external stimuli can be associated to explicit and conscious reactions or to implicit outputs, such as variations in autonomic nervous system (ANS) dynamics [1]. Concerning olfactory elicitation, even if previous studies found changes in electrodermal activity [2] and heart rate [3] associated to different emotional stimuli, the link between self-reported ratings of pleasantness or emotion arousal and variations in ANS dynamics remains partly unclear. Investigating heart rate variability (HRV), an almost instantaneous increase of heart rate was reported after unpleasant odors, whereas pleasant odors showed much smaller effects [4].

In the last decades, phase space theory and related approaches have been largely applied to the investigation of cardiovascular dynamics, given the proven nonlinear mechanisms at the basis of sympatho-vagal interaction [5]. Previous findings demonstrated the effectiveness of HRV nonlinear analysis in the discrimination of moods and autonomic responses to emotional stimuli [6], [7].

In this study, we investigated changes in heartbeat dynamics complexity after the olfactory stimulation through three odorants with different levels of valence (one pleasant, one neutral, and one unpleasant). Self-assessment was used to verify that the odorants chosen for the experimental protocol had effectively induced an emotional response to subjects. We studied how the spatial distances of the trajectories described by cardiovascular series are influenced by the exposition to different hedonic odors, applying Distribution Entropy (DistEn) algorithm [8], [9]. This metrics allows to quantify physiological system complexity even at the single scale, and is useful for the application to ultra-short series. A brief description of the experimental protocol, signal

\footnotetext{
${ }^{1}$ Bioengineering and Robotics Research Centre E. Piaggio \& Department of Information Engineering, University of Pisa, Italy

${ }^{2}$ Department of Chemistry and Industrial Chemistry, University of Pisa, Italy

${ }^{*}$ Corresponding author e-mail: m.nardelli@ing.unipi.it

The research leading to these results has received partial funding from the Italian Ministry of Education and Research (MIUR) in the framework of the CrossLab project (Departments of Excellence).

978-1-7281-5751-1/20/\$31.00 @2020 IEEE
}

processing methodology, along with results and discussion, follows below.

\section{MATERIALS AND METHODS}

\section{A. Subject recruitment and experimental protocol}

Thirty-two participants (aged 26 $\pm 2,16$ males) gave their informed consent to take part in the study, self-reporting no history of clinical cardiovascular and mental diseases [10]. The study was approved by the Ethical Committee of the University of Pisa. Participants were selected in order to present a similar olfactory threshold to n-butanol, normally used to identify the olfactory threshold level, as follows:

- a mother solution (4.05 g/L) of n-butanol was diluted to prepare 10 solutions, with dilution factor chosen as powers of 2, from 8 to 4096;

- each subject was asked to recognize n-butanol solution from distilled water, starting from the lowest concentration and increasing until the odour recognition;

- the subject was selected if the dilution factor of the recognized solution was in the range 256-1024.

During the experiment, subjects were seated and wore earplugs to prevent any auditory cues. Three olfactory stimuli, one pleasant $\left(\mathrm{Od}_{p}\right)$, one neutral $\left(\mathrm{Od}_{n}\right)$, and one unpleasant $\left(\mathrm{Od}_{u}\right)$, were chosen from the literature [11] and used in the experiment:

- $\mathrm{Od}_{p}$ : Benzaldehyde (pleasant bitter almond smell, $106.12 \mathrm{~g} / \mathrm{mol}$ );

- $\mathrm{Od}_{n}$ : n-Butanol (74.12 g/mol);

- $\mathrm{Od}_{u}$ : Isovaleric acid (unpleasant sweat odour, 102.13 $\mathrm{g} / \mathrm{mol}$ ).

For each participant, the order of the presented stimuli was randomized. At the beginning and at the end of the experimental protocol, subjects were in resting state for 3 minutes. Each olfactory stimulus lasted 5 seconds and was presented between two one-minute rest sessions (pre- and post-stimulus sessions). After each post-stimulus session, subjects were asked to assess the elicitation using one score referred to arousal dimension (i.e. the intensity of the evoked emotion, ranged from 1 to 5) and one score related to the valence (i.e. pleasantness/unpleasantness of the stimulus, ranged from -2 to +2 ), in accordance with Russell's model of affect [12]. The ECG signal was continuously acquired using the ECG100C Electrocardiogram Amplifier from BIOPAC inc., with a sampling rate of $500 \mathrm{~Hz}$. The interbeat (RR) series were extracted using the Pan-Tompkins algorithm. Artifacts removal was processed through the use of Kubios HRV software. 


\section{B. Distribution Entropy (DistEn)}

DistEn is a measure of complexity based on the empirical probability distribution of the distances of the phase space trajectories [8], [9]. Given a time series of $\mathrm{N}$ samples $\{u(i), 1 \leq i \leq N\}$ and considering the embedding dimension $m$, the $(N-m)$ points $X_{i}^{m}$ in the reconstructed phase-space have the following coordinates: $X_{i}^{m}=u(i), u(i+1), \ldots, u(i+m-1), 1 \leq i \leq N-m$. The Chebyshev distance $d_{i j}$ between each vector $X i^{m}$ and every vector $X_{j}^{m}$ is computed and the distance matrix $D$ is constructed using all the distance values obtained [8]. The elements of $D$ are then divided into $B$ bins and the probability distribution of the corresponding histogram is obtained, as follows: $p_{t}=\frac{\text { count in bin } \mathrm{t}}{\text { total number of elements in matrix D }}$. The DistEn of $u(i)$ is computed by normalizing the classical Shannon Entropy:

$$
\operatorname{DistEn}(m, B)=-\frac{1}{\log _{2}(B)} \sum_{t=1}^{B} p_{t} \log _{2}\left(p_{t}\right)
$$

The DistEn algorithm was applied to the RR series corresponding to each pre- and post-stimuli session of one minute, using $\mathrm{B}=512$ and $\mathrm{m}=2$.

\section{Statistical analysis}

Since data had non-gaussian distributions, Friedman nonparametric statistical test was used to compare the selfassessment manikin (SAM) scores and DistEn values related to the three stimuli, and Wilcoxon test with Bonferroni correction was applied as post-hoc analysis.

\section{RESULTS}

Concerning SAM values, valence scores assigned to the three odorants resulted to be statistical different after the Friedman test $\left(\mathrm{p}\right.$-value $\left.<10^{-3}\right)$. Specifically, the valence scores related to $\mathrm{Od}_{p}$ were significantly higher than the scores referred to $\operatorname{Od}_{n}$ and $\operatorname{Od}_{u}$ (p-value $<10^{-3}$ ).

Comparing DistEn values related to the three odorants, we used as input of the statistical tests the difference between DistEn computed in the post-stimulus session and DistEn corresponding to the pre-stimulus session. Figure 1 shows the increase of complexity after the unpleasant olfactory stimulation and the decrease after the elicitation through $\mathrm{Od}_{p}$, whereas DistEn values remained almost stable after neutral odor. The effects of the three odorants on cardiovascular complexity resulted to be different after Friedman test (pvalue $=0.018$ ), and $\operatorname{Od}_{p}$ was found to be significantly different from $\mathrm{Od}_{u}(\mathrm{p}<0.05)$ after Bonferroni correction.

\section{DisCUSSION AND CONCLUSION}

This study reports on the variations in ANS complex dynamics after elicitation through hedonic olfactory stimuli. We verified that the three odorants used in the experiments were associated with statistically different levels of valence ( $\mathrm{p}<10^{-3}$ after the Friedman test), in order to be identifiable with positive, neutral, and negative stimuli.

DistEn algorithm was applied to one-minute RR series acquired in the post- and pre-stimulus elicitation sessions. A statistically significant difference in the variation of complexity values was found after the elicitation through $\operatorname{Od}_{u}$

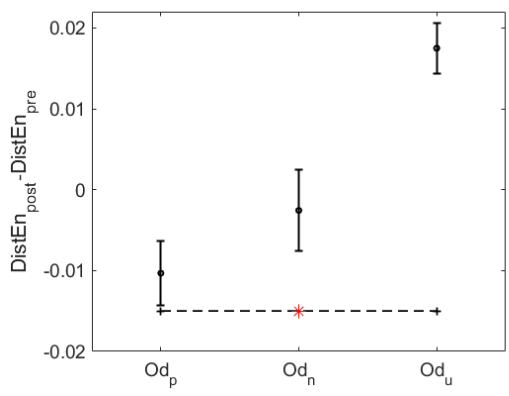

Fig. 1. Median and median absolute deviation (MAD) values of DistEn $\mathrm{n}_{\text {post }}$ - DistEn $n_{\text {pre }}$, for the three odorants $\mathrm{Od}_{p}, \mathrm{Od}_{n}, \mathrm{Od}_{u}$. Asterisk indicates significant statistical differences after post-hoc comparison $(\mathrm{p}<0.05)$

when compared to $O d_{p}$. Specifically, a significant increase was found after the unpleasant stimulus and a decrease was reported after the pleasant odor $(\mathrm{p}<0.05)$.

Previous studies showed that olfaction can be impaired in conditions of chronic stress and mood disturbances [13], and odor properties can induce changes in cognition [14]. This modulation of the ANS system complexity according to the valence of smells can be considered a promising biomarker to test the effectiveness of psychotherapeutic treatments based on hedonic olfactory stimulation.

\section{REFERENCES}

[1] M. Bensafi et al., "Autonomic nervous system responses to odours: the role of pleasantness and arousal," Chemical Senses, vol. 27, no. 8, pp. 703-709, 2002.

[2] C. Van Toller et al., "Skin conductance and subjective assessments associated with the odour of 5- $\alpha$-androstan-3-one," Biological Psychology, vol. 16, no. 1-2, pp. 85-107, 1983.

[3] P. Brauchli et al., "Electrocortical and autonomic alteration by administration of a pleasant and an unpleasant odor," Chemical Senses, vol. 20, no. 5, pp. 505-515, 1995.

[4] W. He et al., "Dynamics of autonomic nervous system responses and facial expressions to odors," Frontiers in psychology, vol. 5, p. 110 , 2014

[5] K. Sunagawa et al., "Dynamic nonlinear vago-sympathetic interaction in regulating heart rate," Heart and vessels, vol. 13, no. 4, pp. 157174, 1998.

[6] M. Nardelli et al., "Heartbeat complexity modulation in bipolar disorder during daytime and nighttime," Scientific reports, vol. 7, no. 1, p. $17920,2017$.

[7] — "Reliability of lagged poincaré plot parameters in ultrashort heart rate variability series: Application on affective sounds," IEEE journal of biomedical and health informatics, vol. 22, no. 3, pp. 741749, 2017.

[8] P. Li et al., "Assessing the complexity of short-term heartbeat interval series by distribution entropy," Medical \& biological engineering \& computing, vol. 53, no. 1, pp. 77-87, 2015.

[9] M. Nardelli et al., "Cardiovascular assessment of supportive doctorpatient communication using multi-scale and multi-lag analysis of heartbeat dynamics," Medical \& biological engineering \& computing, vol. 57, no. 1, pp. 123-134, 2019.

[10] - "Quantifying the lagged poincare plot geometry of ultrashort heart rate variability series: automatic recognition of odor hedonic tone," Medical \& biological engineering \& computing, 2020.

[11] M. Naudin et al., "State and trait olfactory markers of major depression," PLoS One, vol. 7, no. 10, p. e46938, 2012.

[12] J. A. Russell, "A circumplex model of affect." Journal of personality and social psychology, vol. 39, no. 6, p. 1161, 1980.

[13] S. Lombion-Pouthier et al., "Odor perception in patients with mood disorders," Journal of affective disorders, vol. 90, no. 2-3, pp. 187 191, 2006.

[14] A. J. Johnson, "Cognitive facilitation following intentional odor exposure,” Sensors, vol. 11, no. 5, pp. 5469-5488, 2011. 